10

\title{
Магнитоэлектроакустическая динамика в стрейнтронной ячейке памяти с произвольным доступом
}

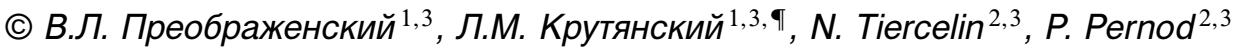 \\ ${ }^{1}$ Институт общей физики им. А.М. Прохорова РАН, Москва, Россия \\ ${ }^{2}$ Univ. Lille, CNRS, Centrale Lille, ISEN, Univ. Valenciennes, UMR 8520 - IEMN, Lille, France \\ ${ }^{3}$ Joint International Laboratory LIA LICS \\ ฯ E-mail: leonid.krut@kapella.gpi.ru
}

Поступило в Редакцию 30 сентября 2019 г.

В окончательной редакции 30 сентября 2019 г.

Принято к публикации 7 октября 2019 г.

Стрейнтронный принцип энергонезависимой магнитоэлектрической памяти с произвольным доступом (MELRAM) привлекает к себе внимание благодаря перспективе достижения на его основе ультранизкого энергопотребления в запоминающих устройствах. Механизм переключения магнитных моментов импульсным деформированием упруго связанных магнитной и пьезоэлектрической подсистем сопряжен с возбуждением акустических колебаний в ячейках памяти. Период колебаний в наноразмерных ячейках сопоставим с временем переключения магнитных моментов, что может вносить искажения в процесс записи информации. Исследуется влияние акустических возбуждений на динамику магнитных переключений с помощью численного моделирования применительно к магнитострикционной ячейке размером $50 \times 50 \times 400 \mathrm{~nm}$ на пьезоэлектрической подложке PMN-PT $\langle 011\rangle$. Определены параметры управляющих электрических импульсов, обеспечивающие устойчивое бинарное переключение магнитных состояний системы.

Ключевые слова: пьезоэлектрик-магнетик, импульсное деформирование, акустические колебания, переключения намагниченности.

DOI: 10.21883/PJTF.2020.01.48864.18056

Снижение энергозатрат на запись и считывание информации является одной из ключевых задач создания нового поколения устройств памяти с произвольным доступом [1]. Перспективным подходом к решению задачи представляется использование магнитоэлектрического взаимодействия в естественных мультиферроиках [2-5] или искусственных мультиферроидных структурах на основе упруго связанных магнитострикционных и пьезоэлектрических компонентов [6-10]. Управление магнитными состояниями с помощью электрических полей в магнитоэлектрических ячейках памяти (MELRAM) позволяет рассчитывать на снижение энергопотребления на запись информации до десятков аттоджоулей на бит [9]. При этом времена переключения, определяемые частотой магнитного резонанса и параметрами спиновой релаксации, оцениваются на уровне долей наносекунды. Пьезоэлектрические деформации в ячейках MELRAM обычно рассматриваются в квазистатическом приближении без учета гиперзвуковых колебаний, возбуждаемых управляющими импульсными электрическими полями. В то же время переменные деформации вносят вклад в эффективные магнитострикционные поля, переключающие магнитные состояния, и могут вносить ошибки в процессы записи. Роль колебаний особенно существенна, если полоса частот управляющих импульсов захватывает частоты собственных акустических мод структуры. В настоящей работе упругая динамика структуры включена в моделирование процессов магнитоэлектрического переключения намагниченности в наномасштабной ячейке MELRAM, управляемой наносекундными и субнаносекундными импульсами электрического напряжения. C помощью программного пакета COMSOL Multiphysics выполнен расчет упругих колебаний структуры, результаты которого использованы для описания процессов переключения намагниченности при численном интегрировании уравнения Ландау-Лифшица-Гильберта.

Геометрия рассматриваемой структуры, представленная на рис. 1, аналогична предложенной в работе [11] для полностью магнитоэлектрической ячейки, в которой и запись, и считывание информации осуществляются с помощью импульсных электрических полей. Размер пьезоэлектрической части ячейки $75 \times 500 \times 200 \mathrm{~nm}$. Роль носителя информации выполняет нанесенная сверху на пьезоэлектрик интерметаллическая магнитострикционная пленка размером $50 \times 50 \times 400 \mathrm{~nm}$ с одноосной магнитной анизотропией, помещенная в магнитное поле $\mathbf{H}$, ориентированное под углом $45^{\circ}$ к оси $y$ в плоскости пленки. Ориентация оси легкого намагничивания в плоскости выбирается таким образом, чтобы обеспечить равенство энергий двух устойчивых равновесных состояний „,“ или „““ с направлениями намагниченности вдоль оси $x$ или $y$ соответственно. При этом угол $\alpha$ между легкой осью и осью $x$ с учетом анизотропии формы определяется отношением поперечного размагничивающего поля к полю магнитной анизотропии: $\cos 2 \alpha=H_{m} / H_{A}$. Равновесные ориентации 


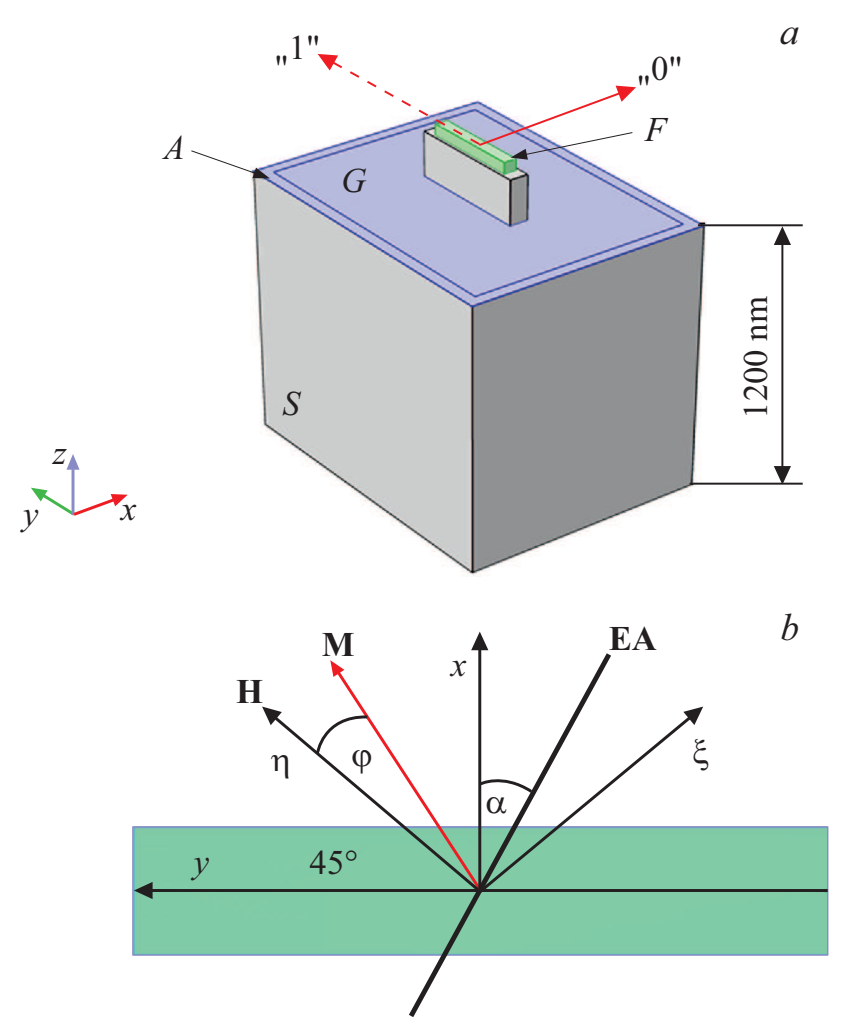

Pис. 1. Модель ячейки MELRAM на подложке PMN-PT $\langle 011\rangle . a-$ геометрия ячейки. $S-$ подложка с расположенной на ней активной ячейкой $\mathrm{PMN}-\mathrm{PT}, F-$ магнитострикционная интерметаллическая пленка, $G$ - электрод, $A-$ виртуальный звукопоглощающий слой. Указаны направления намагниченности, соответствующие состояниям „,“ и „1“. $b-$ взаимная ориентация „легкой оси“магнитной анизотропии ЕА, векторов намагниченности $\mathbf{M}$ и намагничивающего поля $\mathbf{H}$ в плоскости магнитострикционной пленки.

намагниченности вдоль осей $x$ и $y$ достигаются при условии $H=H_{A}^{e f f} / \sqrt{2}$, где эффективное поле магнитной анизотропии равно $H_{A}^{e f f}=H_{A} \sqrt{1-\left(H_{m} / H_{A}\right)^{2}}$.

Пьезоэлектрическая подложка представлена в расчетах фрагментом размером $1000 \times 1300 \times 1200 \mathrm{~nm}$. Во избежание паразитных ревербераций выделенный фрагмент в численной модели окружен по периметру звукопоглощающим слоем. Интерметаллический магнетик помимо основной функции носителя информации играет также роль верхнего электрода. Нижний электрод располагается на подложке вокруг ячейки. Импульсное напряжение, прикладываемое к электродам, создает в структуре анизотропную деформацию $\left\langle u_{x x}-u_{y y}\right\rangle$, управляющую переключениями намагниченности. Полярность напряжения однозначно определяет результирующую ориентацию намагниченности „, $0^{“}$ или „1“ независимо от исходного магнитного состояния.

На рис. 2 представлены полученные с помощью пакета COMSOL Multiphysics результаты расчета деформации $\left\langle u_{x x}-u_{y y}\right\rangle$, усредненной по объему магнитострикционной пленки. Модуль Юнга, коэффициент
Пуассона и плотность пленки приняты в расчете равными $E=126 \mathrm{GPa}, v=0.345$ и $\rho=9600 \mathrm{~kg} / \mathrm{m}^{3}$ соответственно. В качестве пьезоэлектрика рассматривается кристалл $\mathrm{Pb}\left(\mathrm{Mg}_{1 / 3} \mathrm{Nb}_{2 / 3}\right) \mathrm{O}_{3}-\mathrm{PbTiO}_{3}$ (PMN-PT) среза $\langle 011\rangle$, полный набор параметров которого приведен в работе [12]. Расчет выполнен для акустической добротности материалов $Q=1000$. Амплитуда импульсов напряжения принята равной $U_{i}=0.2 \mathrm{~V}$. Результаты, представленные на рис. $2, a$ и $b$, получены для длительностей электрического импульса (по уровню 0.5 от максимума), равных $t_{i}=0.27$ и $1.96 \mathrm{~ns}$ соответственно при одинаковой длительности фронта $0.25 \mathrm{~ns}$. На рисунках видны осцилляции, обусловленные резонансными акустическими колебаниями в ячейке. Частота осцилляций не изменяется при удалении подложки из модели. Излучение акустических волн в подложку увеличивает затухание осцилляций. Видно, что амплитуда второго (обратного) полупериода осцилляций на рис. $2, a, b$ составляет около $45 \%$ от среднего значения во время действия импульса напряжения.
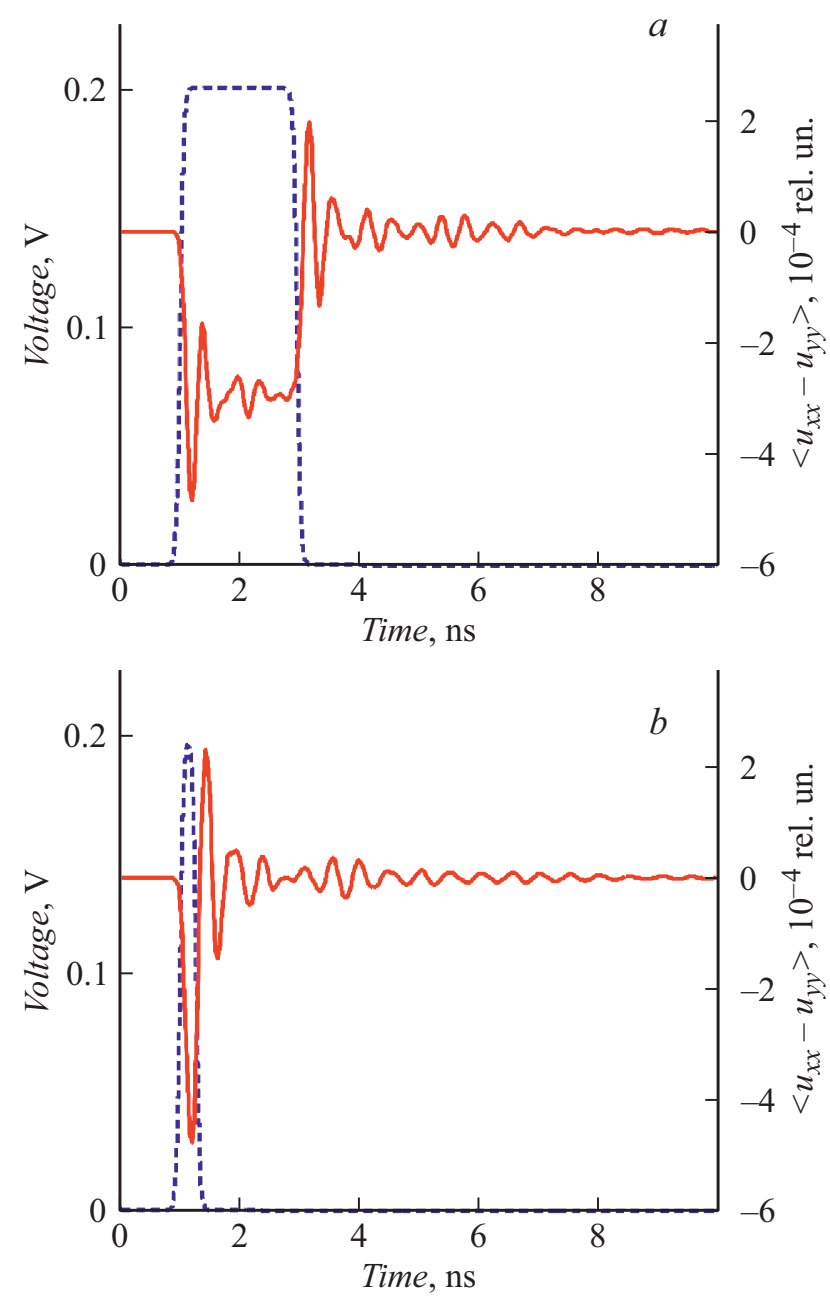

Рис. 2. Зависимость от времени средних по объему пленки деформаций $\left\langle u_{x x}-u_{y y}\right\rangle$ (сплошные линии) для импульсов управляющих напряжений амплитудой $U_{i}=0.2 \mathrm{~V}$ и длительностью $t_{i}=1.96(a)$ и $0.27 \mathrm{~ns}(b)$ (штриховые линии). 
Данные расчета деформаций использованы для описания процесса переключения намагниченности с помощью уравнения Ландау-Лифшица-Гильберта

$$
\frac{\partial \mathbf{M}}{\partial t}=-\gamma \mathbf{T}+\frac{\delta}{M}\left[\mathbf{M} \times \frac{\partial \mathbf{M}}{\partial t}\right]
$$

где $T$ - вращающий момент, $\gamma$ - магнитомеханическое отношение, $\delta$ - коэффициент затухания. В угловых переменных, определяющих проекции магнитного момента в системе координат $(\boldsymbol{\xi}, \boldsymbol{\eta}, \boldsymbol{\xi})$, связанной с направлением намагничивающего поля $\boldsymbol{\eta}\|\mathbf{H}, \boldsymbol{\xi}\| \mathbf{z}, M_{\xi}=M \cos \theta \sin \varphi$, $M_{\eta}=M \cos \theta \cos \varphi, M_{\xi}=M \sin \theta$, где угол $\theta$ отсчитывается от плоскости пленки, уравнение (1) преобразуется к виду

$$
\begin{gathered}
\frac{\partial \varphi}{\partial t}=-\frac{\gamma}{M \Delta}\left[T_{\xi} \cos \theta+T_{\xi}(\sin \theta \sin \varphi-\delta \cos \varphi)\right], \\
\frac{\partial \theta}{\partial t}=-\frac{\gamma}{M \Delta}\left[T_{\xi} \delta \cos ^{2} \theta+T_{\xi}(\cos \theta \cos \varphi\right. \\
+\delta \sin \theta \cos \theta \sin \varphi)],
\end{gathered}
$$

где $\Delta=\left(1+\delta^{2}\right) \cos ^{2} \theta \cos \varphi$,

$$
\begin{aligned}
T_{\xi} / M= & \frac{1}{4}\left(H_{A}^{e f f}-H_{A}-H_{m}\right) \sin 2 \theta \cos \varphi-H \sin \theta \\
+ & \frac{B}{2 M}\left\langle u_{x x}-u_{y y}\right\rangle \sin 2 \theta \sin \varphi \\
T_{\xi} / M= & -\frac{1}{2} H_{A}^{e f f} \cos ^{2} \theta \sin 2 \varphi+H \cos \theta \sin \varphi \\
& +\frac{B}{M}\left\langle u_{x x}-u_{y y}\right\rangle \cos ^{2} \theta \cos 2 \varphi .
\end{aligned}
$$

Здесь $B-$ постоянная магнитострикции.

На рис. $3, a, b$ представлены результаты решения уравнений (2) с переменными деформациями $\left\langle u_{x x}-u_{y y}\right\rangle$, приведенными на рис. $2, a$ и $b$ соответственно. В качестве начального равновесного состояния принято $\varphi=\pi / 4$ и $\theta=0$. Расчет выполнялся применительно к наноструктурированной пленке $N\left(\mathrm{TbFe}_{2} / \mathrm{FeCo}\right)$ $(N-$ количество бислоев) с гигантской магнитострикцией $(B=10 \mathrm{MPa})$ и магнитными параметрами $M=200 \mathrm{emu} / \mathrm{cm}^{3}, H_{A}=1.3 \mathrm{kOe}, \delta=0.15$. Вставки на рис. $3, a, b$ демонстрируют фазовые портреты колебаний намагниченности $\theta(\varphi)$.

По данным расчета переключение намагниченности реализуется при длительности импульса электрического напряжения больше $t_{i} \cong 0.32 \mathrm{~ns}$, тогда как время магнитной релаксации составляет около $1 \mathrm{~ns}$. Отметим, что для квазистатического приближения, не учитывающего акустических возбуждений, расчет дает значение минимальной длительности управляющего импульса $t_{i}=0.25 \mathrm{~ns}$ при той же амплитуде $U_{i}=0.2 \mathrm{~V}$. Для длительности $t_{i}=0.27 \mathrm{~ns}$ расчет демонстрирует динамическое отклонение намагниченности от ориентации „,“ с последующей релаксацией в исходное состояние (рис. $3, b$ ).
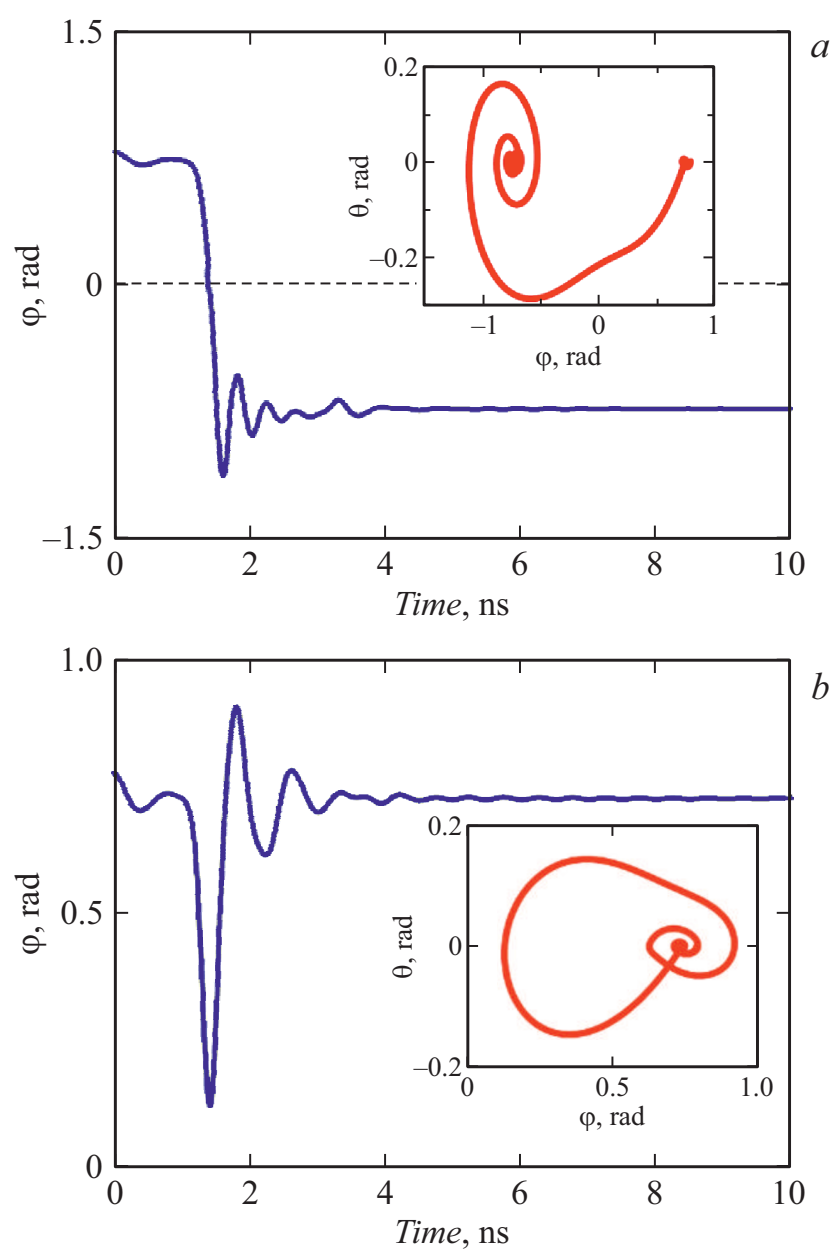

Рис. 3. Зависимость от времени угла $\varphi$ между магнитным моментом и намагничивающим полем при длительностях управляющих импульсов $t_{i}=1.96(a)$ и $0.27 \mathrm{~ns}(b)$. На вставках приведены фазовые портреты $\theta(\varphi)$ движения намагниченности в режиме переключения $(a)$ и в отсутствие переключения $(b)$ магнитных состояний.

Пример переключения „0“-, „1“ для $t_{i}=1.96 \mathrm{~ns}$ и $U_{i}=0.2 \mathrm{~V}$ приведен на рис. 3, $a$. Минимальная амплитуда напряжения, обеспечивающая переключение намагниченности при данной длительности импульса, составляет $U_{i}=70 \mathrm{mV}$ в отличие от $U_{i}=46 \mathrm{mV}$ в квазистатическом приближении. При напряжении противоположного знака переключение отсутствует для любых длительностей импульса, что подтверждает однозначную зависимость конечного состояния магнитной системы от полярности управляющего электрического поля.

Моделирование процесса переключения магнитных состояний в магнитоэлектрической ячейке MELRAM выполнено на примере структуры, демонстрирующей явно выраженные резонансные возбуждения акустических колебаний, сопровождающих приложенные импульсные электрические поля. Колебания накладывают дополнительные ограничения на минимальную амплитуду и длительность управляющих импульсов по сравнению с 
результатами квазистатического приближения. Несмотря на значительные амплитуды переменных составляющих деформаций при субнаносекундных электрических напряжениях, надлежащий выбор параметров управляющих импульсов обеспечивает устойчивое переключение магнитных состояний в стрейнтронной ячейке.

\section{Финансирование работы}

Работа выполнена при финансовой поддержке Российского фонда фундаментальных исследований (грант № 16-29-14022).

\section{Конфликт интересов}

Авторы заявляют, что у них нет конфликта интересов.

\section{Список литературы}

[1] Nanomagnetic and spintronic devices for energy-efficient memory and computing / Eds J. Atulasimha, S. Bandyopadhyay. 1st ed. John Wiley \& Sons, Ltd, 2016. $359 \mathrm{p}$.

[2] Chen X., Hochstrat A., Borisov P., Kleemann W. // Appl. Phys. Lett. 2006. V. 89. N 20. P. 202508 (1-3).

[3] Bibes M., Barthélémy A. // Nature Mater. 2008. V. 7. N 6. P. 425-426.

[4] He X., Wang Y., Wu N., Caruso A.N., Vescovo E., Belashchenko K.D., Dowben P.A., Binek C. // Nature Mater. 2010. V. 9. N 7. P. 579-585.

[5] Берзин А.А., Винокуров Д.Л., Морозов А.И. // ФТТ. 2016. T. 58. B. 11. C. 2237-2241.

[6] Tiercelin N., Dusch Y., Klimov A., Giordano S., Preobrazhensky V., Pernod P. // Appl. Phys. Lett. 2011. V. 99. N 19. P. 192507. doi.org/10.1063/1.3660259

[7] Dusch Y., Tiercelin N., Klimov A., Giordano S., Preobrazhensky V., Pernod P. // J. Appl. Phys. 2013. V. 113. N 17. P. 17C719. DOI: $10.1063 / 1.4795440$

[8] Ahmad H., Atulasimha J., Bandyopadhyay S. // Sci. Rep. 2015. V. 5. P. 18264 . DOI: $10.1038 /$ srep 18264

[9] Giordano S., Dusch Y., Tiercelin N., Pernod P., Preobrazhensky V. // Phys. Rev. B. 2012. V. 85. N 15. P. 155321.

[10] Camsari K.Y., Faria R., Hassan O., Sutton B.M., Datta S. // Phys. Rev. Appl. 2018. V. 9. N 4. P. 044020.

[11] Preobrazhensky V., Krutyansky L., Tiercelin N., Dusch Y., Sigov A., Pernod P., Giordano S. // Ferroelectrics. 2018. V. 532. N 1. P. 160-167. DOI: $10.1080 / 00150193.2018 .1499403$

[12] Wang F., Luo L., Zhao D.Z.X., Luo H. // Appl. Phys. Lett. 2007. V. 90. N 21. P. 212903. 